\title{
A Spectroscopic Study on the Nonlinear Optical Susceptibilities of Organic Molecules
}

\author{
S. Foerier ${ }^{a, *}$, V.K. VAlev ${ }^{a}$, G. Koeckelberghs ${ }^{a}$, \\ I.A. KolmycheK ${ }^{b}$, O.A. Aksipetrov ${ }^{b}$ And T. Verbiest ${ }^{a}$ \\ ${ }^{a}$ Institute for Nanoscale Physics and Chemistry (INPAC) \\ Molecular and Nanomaterials
}

Katholieke Universiteit Leuven, Celestijnenlaan 200 D, 3001 Leuven, Belgium

${ }^{b}$ Department of Physics, Moscow State University, 119992 Moscow, Russia

We report on the investigation of the spectral dependence of nonlinear optical susceptibilities, that describe second harmonic generation - circular dichroism, the nonlinear optical analogue of circular dichroism. The design and implementation of a state of the art experimental setup, used for the characterization of a series of organic molecules in the near-infrared range, will be discussed. We emphasize on the main characteristics like wavelength control, polarization and data-acquisition to present the setup. Some preliminary results show its strength to detect chirality at the molecular and supramolecular level.

PACS numbers: 42.65.--k, 78.20.--e

\section{Introduction}

Since their discovery in 1962 [1], conjugated polymers have opened up an exciting new field of research. Nowadays they are widely used in mobile phones, thin film transistors, solar cells and many other applications [2]. Furthermore, from the point of view of fundamental science, they possess very interesting electrical and optical properties due to their extended aromaticity. Prominent among those is the well known effect of chiral stacking, analogue to the one in liquid crystals [3].

Highly sensitive to chirality, second harmonic generation (SHG) [4] has been shown to be a valuable detection tool in both industry and academics. The applications range from determination of the first-order nonlinear susceptibilities, over biological imaging [5] to the characterization of oriented surfaces and interfaces. Together with the introduction of new laser sources, SHG has allowed for an unprecedented set of highly sensitive characterization methods. However, due

${ }^{*}$ corresponding author; e-mail: stijn.foerier@fys.kuleuven.be 
to the fact that lasers possess an inherent monochromaticity, their use is somewhat limited in the sense that the spectral dependence of various parameters cannot be measured. This problem has been solved partially by using, for example, Ti:sapphire femtosecond lasers with a wide wavelength range. Nevertheless, the wavelength span of even those lasers is restricted on the near-infrared part of the spectrum. With the advent of the optical parametric amplifier (OPA), the issue appears to have found a better answer. OPAs offer a broad continuum of available wavelengths in the region of 1100-1600 nm. Furthermore, by using harmonic generators it is possible to extend the range to include the visible and the near UV.

In this report we will discuss the design and implementation of a state of the art experimental instrument which, benefiting from the capabilities of the OPA, enables us to perform a complete characterization of the nonlinear optical properties of various organic systems over a large wavelength range and with very high sensitivity. This instrument will provide valuable insight concerning (some) aspects of the optical, magnetic and electrical properties of these molecules, especially for in- and off-resonant conditions. After addressing briefly the fundamental theoretical aspects of SHG, circular dichroism (CD) and their combination, we will describe extensively the experimental setup. An emphasize will be made on the broad available wavelength range, the precise polarization control and the highly sensitive detection scheme. Finally we will present our first results, which appear quite promising, although still preliminary.

\section{Theoretical introduction}

SHG has been known for a few decades already. The process describes the interaction of light with matter in the second order, and the nonlinear polarization associated with it can be expressed in the following manner [6]:

$$
\boldsymbol{P}_{\mathrm{NL}}=\chi^{(2), e e e} \boldsymbol{E} \boldsymbol{E},
$$

where $\chi^{(2), \text { eee }}$ is the second order susceptibility and $\boldsymbol{E}$ - the electric field component of the incident light. It should be noted that this equation is only valid in the electric-dipole approximation. Indeed, previous research has shown that, for instance in polythiophenes, magnetic-dipole contributions can be significant and therefore should not be neglected [7]. Hence, the nonlinear polarization and magnetization are given by

$$
\begin{aligned}
& \boldsymbol{P}_{\mathrm{NL}}=\chi^{(2), \text { eee }} \boldsymbol{E} \boldsymbol{E}+\chi^{(2), e e m} \boldsymbol{E} \boldsymbol{B}, \\
& \boldsymbol{M}_{\mathrm{NL}}=\chi^{(2), m e e} \boldsymbol{E} \boldsymbol{E} .
\end{aligned}
$$

One of the most intriguing phenomena in nonlinear optics is the existence of the nonlinear analogue of circular dichroism, where chiral molecules show a different efficiency for the generation of right and left circularly polarized second-harmonic light. Moreover, SHG-CD has been shown to be orders of magnitude larger than linear CD and therefore constitutes a much more sensitive probing tool. In Table I, the nonvanishing susceptibility tensor components for isotropic chiral and achiral surfaces are shown. 
TABLE I

Nonvanishing tensor components $\chi_{i j k}^{(2), e e e}, \chi_{i j k}^{(2), m e e}$, and $\chi_{i j k}^{(2), e e m}$ for isotropic chiral and achiral surfaces, where $i, j$, and $k$ are the Cartesian coordinates on the sample.

\begin{tabular}{c|l|l}
\hline \hline Tensor & $\begin{array}{l}\text { Achiral components for all isotropic } \\
\text { surfaces }\end{array}$ & $\begin{array}{l}\text { Additional components for chiral } \\
\text { surfaces }\end{array}$ \\
\hline$\chi_{i j k}^{(2), e e e}$ & $z z z, z x x=z y y$ & $x y z=x z y=-y x z=-y z x$ \\
& $x x z=x z x=y y z=y z y$ & $z z z, z x x=z y y$, \\
\hline$\chi_{i j k}^{(2), m e e}$ & $x y z=x z y=-y x z=-y z x$ & $x x z=x z x=y y z=y z y$ \\
\hline$\chi_{i j k}^{(2), e e m}$ & $x y z=-y x z, z x y=-z y x$, & $z z z, z x x=z y y, x x z=y y z$, \\
& $x z y=-y z x$ & $x z x=y z y$
\end{tabular}

The electric field component of the radiation with the nonlinear polarization and magnetization as a source can be written as

$$
\boldsymbol{E}_{i}(2 \omega)=f_{i} \boldsymbol{E}_{\mathrm{P}}^{2}(\omega)+g_{i} \boldsymbol{E}_{\mathrm{S}}^{2}(\omega)+h_{i} \boldsymbol{E}_{\mathrm{P}}^{2}(\omega) \boldsymbol{E}_{\mathrm{S}}(\omega)
$$

where $f_{i}, g_{i}, h_{i}$ are linear combinations of the tensor components of the nonlinear susceptibilities $\chi_{i j k}^{(2), e e e}, \chi_{i j k}^{(2), m e e}$ and $\chi_{i j k}^{(2), e e m}$ and $\boldsymbol{E}_{\mathrm{S}}(\omega)$ and $\boldsymbol{E}_{\mathrm{P}}(\omega)$ are the $s$ - and $p$-polarization components of the fundamental light. Subscript $i$ refers to the $s$ and $p$-polarized components of the second-harmonic field. The exact written form of these coefficients depends on the symmetry of the sample and the contributions that are included in the theory.

\section{Experimental setup}

The laser source used in all the experiments is a tuneable, amplified femtosecond system, comprising four distinct lasers. These include a seed-laser (Mai Tai, Spectra Physics - Newport) that provides femtosecond pulses (120 fs) at $800 \mathrm{~nm}$ with a repetition rate of $80 \mathrm{MHz}$. The pump-laser (Empower, Spectra Physics - Newport) is a Nd:YLF laser, producing $10 \mathrm{~W}$ at $527 \mathrm{~nm}$. The seed-pulses are amplified in the Spitfire (Spectra Physics - Newport) to produce femtosecond pulses (120 fs, $1 \mathrm{kHz}, 800 \mathrm{~nm}$ ) with $1 \mathrm{~mJ}$ of energy per pulse. Afterwards the light is sent to an OPA (Spectra Physics - Newport) that changes the wavelength from $800 \mathrm{~nm}$ to any specific value between 1140 and $1570 \mathrm{~nm}$. This chain of lasers is extremely sensitive to atmospheric conditions in the place of its operation and henceforth care should be taken to ensure a stable environment. Our laboratory was provided with temperature regulation equipment operating with a precision of less than $1^{\circ} \mathrm{C}$ and a flow box to ensure a constant, clean ventilation. Additionally, we mounted an extra nitrogen-supply in order to purge the Mai Tai, further stabilizing the output of this seed-laser.

A schematic of the optical setup is shown in Fig. 1. After leaving the OPA, the beam first encounters an adjustable mirror and then passes through a spatial filter that collimates the light. The combination of a half wave plate and a polarizer 
is then used to adjust the intensity. Next, a quarter wave plate enables us to change the input polarization. Further on, a lens with focal length of $20 \mathrm{~cm}$, focuses the beam onto the sample, through a visible light filter which blocks the visible part of spectrum, while transmitting $90 \%$ of the infrared radiation. The sample-holder is mounted on a rotation stage permitting the variation of the angle of incidence with respect to the sample normal. Afterwards, an analyzer enables us to select specific tensor components of the SHG. The subsequent IR-filter and monochromator ensure the detection of the proper wavelength by a photomultiplier (PMT), which is connected to a gated boxcar integrator. Two IR photodiodes are used in the setup to monitor the IR intensity. The first one is placed at the output of the OPA in order to follow the stability of the laser and if necessary to correct laser intensity drift in the obtained results. The second photodiode detects the reflection of the IR-filter and monitors the polarization pattern of the fundamental light as a reference. It can also reveal the occurrence of sample damage.



Fig. 1. Layout of the setup. 1 - Mai Tai; 2 - Empower; 3 - Spitfire; 4 - OPA; M mirror; BS1 - beam splitter; SF — spatial filter; $\lambda / 2$ - half wave plate; $\mathrm{P}$ - polarizer; $\lambda / 4$ - quarter wave plate; L1 — lens; VIS-F — UV/VIS-filter; S - sample; A analyzer; BS2 - beam splitter; IR-F — IR-filter; L2 - lens; MC — monochromator; PMT - photomultiplier; PD1 - photodiode; PD2 - photodiode; FD - frequency doubler; BCI - boxcar integrator; M-CON - motion controller. 
The most important feature of our setup is its flexibility with respect to wavelength (see Eq. (4)). Further emphasizing this point, it should be noted that by placing a mirror behind the Mai Tai (before the Spitfire), we can add the 750$850 \mathrm{~nm}$ wavelength region to our spectrum. However this implies changes in the detection scheme and therefore will not be further discussed in this article. In general, our large wavelength range requires special optics for its implementation. For instance, highly reflective mirrors providing excellent pulse width conservation are available commercially only for specific, and rather narrow, spectral ranges. In Fig. 1, the mirror $\mathrm{M}$ was a compromise; it has a protected gold coating, thereby providing a reflectance above $95 \%$ in the entire near-IR part of the spectrum (650-1700 nm) and, at the same time, its pulse broadening effect is not significant. From our experience, it is very difficult to find high power polarizers that are suitable for use in this wavelength range. Our choice went to a Glan-Thompson polarizer Meadowlark (GTP-M10) without antireflection coating and a broad operation wavelength of $320-3200 \mathrm{~nm}$. This is of particular importance since a good extinction ratio is required not only in the IR-range but also in the visible part of the SHG. Similar considerations guided our choice of the quarter wave plate; Meadowlark's wave plates are available with an appropriate IR-coating and therefore they were found to be suitable. Because of the large wavelength range and the high sensitivity of SHG experiments to focussing conditions, chromatic aberrations from the lens L1 could not be ignored. This issue was addressed by mounting the lens on a translation stage, thereby enabling us to compensate for the variations of focal distance. Furthermore, due to the high peak power in our system, various undesirable nonlinear optical effects can be observed from the optical components. These include the generation of higher harmonics or even white light. Additionally, several of the organic molecules to be studied exhibit fluorescence. By placing a monochromator before our PMT, we were able to troubleshoot all these unwanted effects and, at the same time, to ensure the detection of genuine SHG.

The polarization is another major issue in our studies since it determines the selection of nonlinear susceptibility tensor components (see Eq. (4)). The light that emerges from the OPA is vertically polarized with respect to the optical table. The polarizers present an extinction ratio of $10^{-5}$ over the central $2 / 3 \mathrm{rd}$ part of the clear aperture. We found this to be sufficient since our average power is generally kept low, in order to avoid the unwanted nonlinear optical phenomena mentioned above. Furthermore, rotation of the polarizing components is done with a precision of the order of a mdeg. According to specifications, the wave plates have a retardation accuracy of $\lambda / 100$ over their entire wavelength range and we have found no fault with them. BS1 and BS2 are both nonpolarizing beam splitters.

The data-acquisition is done by the combination of a gated boxcar integrator (Stanford Research Systems, SR250) and a computer program written in LabVIEW. The boxcar integrator is triggered by the output signal of the Spitfire, 
which occurs at the moment the laser pulse leaves the amplifier. The SR250 has an "active baseline subtraction" feature in which every second detected electronic pulse is inverted and added to an average. In their manual, Stanford Research Systems recommend to block every second laser pulse so that the boxcar then measures only ambient noise and effectively subtracts it from the signal. Following this logic, the SR250 is equipped with a voltage output providing a signal at half the trigger frequency that could aliment a laser beam shutter, for instance. This practice is known to improve the signal to noise ratio by an order of magnitude at least. However, it also results in a loss of half the laser pulses. We believe that a better solution is to double the $1 \mathrm{kHz}$ trigger frequency from the Spitfire and thereby we constructed an electronic device that splits the trigger pulse and retards half of it for an adjustable time delay within the trigger period. The loss on the trigger amplitude is negligible and henceforth, we achieve a significantly more sensitive detection scheme.

Through the use of automatic rotation stages (Newport M-URM80CC) and a motion controller (Newport Motion Controller/Driver, ESP300) the computer program allows a routine operation of three experimental degrees of freedom, namely, simultaneous control of the position of the quarter wave plate, the sample position (and thus the angle of incidence) and the position of the analyzer.

Finally, the data from the SHG and the two reference detectors for the fundamental light are written in series of txt-format files and are processed by a combination of analytical Origin-C programs and Lab Talk scripts, written for the Origin software.

\section{Experimental results}

In Fig. 2b, we plotted the SHG and fundamental (at $1140 \mathrm{~nm}$ ) responses of a Langmuir-Blodgett film constituted from a chiral helicene [8], after rotating azimuthally the analyzer, for a fixed incoming polarization. The latter was set at $45^{\circ}$ to the $p$-direction (i.e. $\boldsymbol{E}_{\mathrm{S}}=\boldsymbol{E}_{\mathrm{P}}$ ), thereupon, from Eq. (4), it follows that:

$$
I_{i}(2 \omega)=\left|f_{i}+g_{i}+h_{i}\right|^{2}\left|E_{\mathrm{S}}(\omega)\right|^{4},
$$

where the SH intensity for a certain polarization direction is entirely determined by the combination of nonvanishing susceptibility tensor elements. Strictly speaking, the helicene film has a $C_{2}$-symmetry, however it can be shown that, from the point of view of chirality, the combination of nonvanishing tensor elements is similar to that of an isotropic chiral surface [4]. Therefore, we can compare its nonlinear optical response with that of an isotropic achiral reference sample (see Fig. 2a). The presence of extra chiral components (see Table I) in the sum $f_{i}+g_{i}+h_{i}$ leads to a shift of the SHG polarization pattern with respect to both the achiral SHG and the fundamental responses.

In Table II, it can be seen that this shift diminishes with increasing wavelength, which is in agreement with the trend observed previously with linear CD spectroscopy in the short range 570 to $600 \mathrm{~nm}[8]$. 



Fig. 2. Polarization pattern with fixed input polarization at $45^{\circ}$ to the $p$-direction and rotating analyzer starting from $-45^{\circ}$ for an achiral reference sample (a) and a chiral helicene (b).

\section{TABLE II}

Polarization pattern shift in degrees between chiral and achiral isotropic samples as function of the fundamental wavelength in $\mathrm{nm}$.

\begin{tabular}{c|c|c|c}
\hline \hline Fundamental wavelength & $1140 \mathrm{~nm}$ & $1200 \mathrm{~nm}$ & $1300 \mathrm{~nm}$ \\
\hline Polarization pattern shift & $25.2^{\circ}$ & $9.7^{\circ}$ & $2.3^{\circ}$
\end{tabular}

In a typical SHG-CD experiment, following the polarizer, a quarter wave plate modulates the incoming polarization from linear to circular, for a fixed position of the analyzer, thereby addressing different tensor components of the nonlinear susceptibility.

In Fig. 3, the sample was an isotropic spincoated, $100 \mathrm{~nm}$ thin film of a chiral polythiophene, poly ((S)-3-(3,7-dimethyloctyl)thiophene).

When both the polarizer and the analyzer are set at $p$-polarization (black triangles), the maxima correspond to linearly polarized light (parallel polarizations),

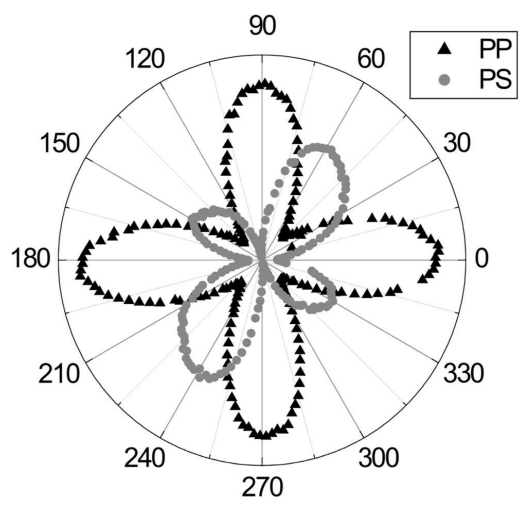

Fig. 3. Polarization pattern for poly ((S)-3-(3,7-dimethyloctyl)thiophene). 
whereas the minima - to circularly polarized (half crossed polarizations). For this geometry, there are no, or extremely weak chiral contributions to the susceptibility since only the $z x x, z y y, z z z$ and $x x z$ tensor elements are addressed, non of which are chiral for the dominant $\chi_{i j k}^{(2), e e e}$ (see Table I). When the analyzer is set to $s$-polarization (gray dots), it can be seen that the minima occur at linearly polarized light (crossed polarizations), whereas the maxima correspond to circular polarization (half crossed polarizations). In this case, the response is a mixture of the nonchiral $y y z$ and the chiral $y x z$ components and the latter leads to a different SHG intensity for right and left circularly polarized light.

These observations are also in good agreement with previously reported data [7] and in the near future we shall investigate their wavelength dependence.

\section{Conclusion}

We reported on the implementation of an entire new setup capable of performing a full, spectral analysis of various organic systems. We discussed all the issues that should be taken into account when implementing such a setup among which polarization and wavelength control are the most prominent. Preliminary results show the possibilities of the setup and that there is a clear spectral dependence regarding SHG-CD.

\section{Acknowledgments}

We acknowledge financial support from the Fund for Scientific Research Flanders (FWO-V) and the University of Leuven (GOA). S.F. acknowledges financial support from the IWT. G.K. is a postdoctoral fellow of the FWO-V. We also would like to thank Thomas J. Katz (Columbia University) for providing us with the helicene sample and Rik Strobbe and Frans Hennau for helping us with the electronics of the SHG setup.

\section{References}

[1] H. Shirakawa, E.J. Louis, A.G. MacDiarmid, C.K. Chiang, A.J. Heeger, J. Chem. Soc. Chem. Commun. 16, 578 (1977).

[2] Handbook of Conducting Polymers, Eds. T.A. Skotheim, R.L. Elsenbaumer, J.V. Reynolds, 2nd ed., Marcel Dekker, New York 1998.

[3] See for example Ch. 6 in: Materials - Chirality, M.M. Green, R.J.M. Nolte, E.W. Meijer, Vol. 24, Topics in Stereochemistry, Wiley-Interscience, New Jersey 2003.

[4] S. Sioncke, T. Verbiest, A. Persoons, Mater. Sci. Eng. R 42, 115 (2003).

[5] P. Campagnola, W.A. Mohler, Opt. Photonics News 14, 40 (2003).

[6] R.W. Boyd, Nonlinear Optics, Academic Press, San Diego 1992.

[7] T. Verbiest, S. Sioncke, G. Koeckelberghs, C. Samyn, A. Persoons, E. Botek, J.M. André, B. Champagne, Chem. Phys. Lett. 404, 112 (2005).

[8] C. Nuckolls, T.J. Katz, G. Katz, P.J. Collings, L. Castellanos, J. Am. Chem. Soc. 121, 79 (1999). 